\title{
Human Body Heat for Powering Wearable Devices: From Thermal Energy to Application
}

\author{
Moritz Thielen $^{\mathrm{a}, 1, *}$, Lukas Sigrist ${ }^{\mathrm{b}, 1}$, Michele Magno $^{\mathrm{c}, \mathrm{d}}$, Christofer Hierold $^{\mathrm{a}}$, \\ Luca Benini ${ }^{\mathrm{c}, \mathrm{d}}$ \\ ${ }^{a}$ Micro- and Nanosystems Group (MNS), ETH Zurich, Switzerland \\ ${ }^{b}$ Computer Engineering and Networks Laboratory (TIK), ETH Zurich, Switzerland \\ ${ }^{c}$ Integrated Systems Laboratory (IIS), ETH Zurich, Switzerland \\ ${ }^{d}$ Dipartimento Elettrica e dell'Informazione (DEI), Università di Bologna, Italy
}

\begin{abstract}
Energy harvesting is the key technology to enable self-sustained wearable devices for the Internet of Things and medical applications. Among various types of harvesting sources such as light, vibration and radio frequency, thermoelectric generators (TEG) are a promising option due to their independence of light conditions or the activity of the wearer. This work investigates scavenging of human body heat and the optimization of the power conversion efficiency from body core to the application. We focus on the critical interaction between thermal harvester and power conditioning circuitry and compare two approaches: (1) a high output voltage, low thermal resistance $\mu \mathrm{TEG}$ combined with a high efficiency actively controlled single inductor DC-DC converter, and (2) a high thermal resistance, low electric resistance mTEG in combination with a lowinput voltage coupled inductors based DC-DC converter. The mTEG approach delivers up to $65 \%$ higher output power per area in a lab setup and 1\% to $15 \%$ in a real-world experiment on the human body depending on physical activity and environmental conditions. Using off-the-shelf and low-cost components, we achieve an average power of $260 \mu \mathrm{W}$ ( $\mu \mathrm{TEG}$ ) to $280 \mu \mathrm{W}$ (mTEG) and power densities of $13 \mu \mathrm{W} \mathrm{cm} \mathrm{cm}^{-2}$ ( $\mu \mathrm{TEG}$ ) to $14 \mu \mathrm{W} \mathrm{cm}{ }^{-2}$ (mTEG) for systems worn on the human wrist. With the small and lightweight harvesters optimized for wearability, $16 \%$ (mTEG) to $24 \%$ ( $\mathrm{TTEG}$ ) of the theoretical maximum efficiency is achieved in a worst-case scenario. This efficiency highly depends on the application specific conditions and requires careful system design. The harvesters can power wearables in different use cases, for example a multi-sensor bracelet that measures activity, acquires images and displays results.
\end{abstract}

\footnotetext{
* Corresponding author.

Email addresses: thielen@micro.mavt.ethz.ch (Moritz Thielen), lukas.sigrist@tik.ee.ethz.ch (Lukas Sigrist), magnom@iis.ee.ethz.ch (Michele Magno), christofer.hierold@micro.mavt.ethz.ch (Christofer Hierold), lbenini@iis.ee.ethz.ch (Luca Benini)

${ }^{1}$ Both authors contributed equally to this work.
}

Preprint submitted to Energy Conversion and Management

October 19, 2016

(C) 2016. This manuscript version is made available under the Elsevier user license http://www.elsevier.com/open-access/userlicense/1.0/ 
Keywords: Energy Harvesting, TEG, Wearable, DC-DC Conversion 2010 MSC: 00-01, 99-00

\section{Introduction}

Recent developments in sensing technology, low power processing and communication have enabled a rapidly emerging field, the Internet of Things (IoT), poised to become the largest electronics market for the semiconductor industry [1. A promising vision is to have billions of sensor devices that are wirelessly connected and can collect and process data to facilitate a wide range of application such as fitness and sports, machinery or health monitoring 2]. The major trend in IoT technology is decreasing of both form factor and power consumption while increasing functionality. A fast growing class of such devices is wearable, where sensors nodes are tightly coupled with the human body [3. Low power consumption is crucial in wearable systems due to the tight weight and size constraints for batteries, which severely limit the energy that can be stored in the device. Although integrated circuits have significantly improved their energy efficiency, battery technology is not tracking at the same speed in terms of volumetric energy density improvements. Additionally, user expectations for wearable devices imply a lifetime in the orders of months, if not years, instead of daily recharges common for contemporary wearables 4]. Hence, ultra-low power design alone is not sufficient to make these devices truly wearable.

Energy harvesting $(\mathrm{EH})$ is an emerging but reasonably mature technology to overcome the limited lifetime of battery-operated wearable devices and allows continuous recharging of the energy storage during use [1, 3]. Wearables are, however, very tightly constrained in terms of size and weight and must also couple with the body. Therefore, the possibilities for EH systems are more restricted than for other applications. Energy can be harvested from various environmental sources [5] including light using photovoltaics [6], movement of the wearer [7, from radio frequency energy (RF) 8] or from temperature differences using thermoelectric generators (TEG) [9, 10. Photovoltaic or RF harvesters limit the application of zero-power wearables to environments where sufficient ambient light or RF emmissions is provided to satisfy the energy budget. Movement-based harvesting systems require an active wearer and usually have unstable power generation characteristics. The human body in contrast is a constant heat source and typically a temperature difference exists between body core and the environment. Even in a scenario where the wearer is stationary and situated in a dark room (e.g. during sleep), energy can be produced 11]. Lower ambient temperatures, the presence of air convection or increased activity of the wearer can drastically increase the amount of accumulated energy [12. Because the voltages produced by thermal harvesting are typically too low to power wearable electronics, a conversion stage (DC-DC) with high conversion efficiency needs to be included into a wearable system. A complete system analysis from body core heat to the application is required to maximize both output power and wearability. 


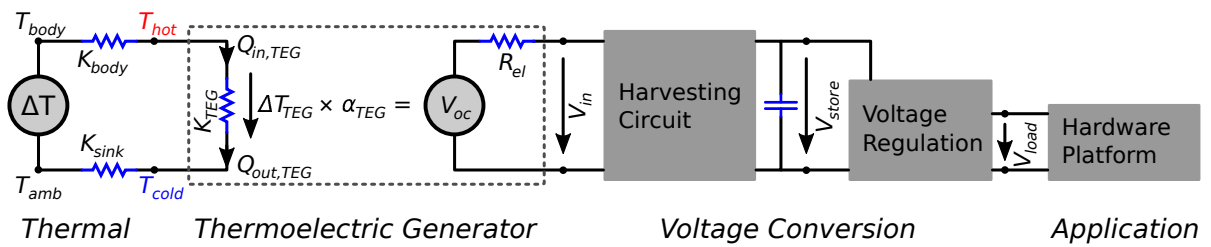

Figure 1: System overview of a TEG harvesting driven wearable application.

Contributions

In this work we report a system analysis and optimization of the complete pathway from human body heat to a wearable hardware platform as shown in Figure 1. This includes the following main objectives:

- Comparison of different TEG approaches for low- $\Delta \mathrm{T}$ applications.

- Characterization and comparison of state-of-the-art voltage conversion architectures.

- Laboratory and real-world characterization of two thermal harvesting systems for the human wrist.

- Case study with a zero-power multi-sensor bracelet to confirm that thermal energy harvesting can be effectively applied in smart wearable devices.

After covering the related work in Section 2, we discuss the different building blocks of a body heat driven wearable application and the interaction between the individual elements. Section 3 discusses thermoelectric energy conversion, classification of TEG, their application on the human body and the harvesters used in this study. In Section 4 two voltage conversion architectures and the voltage regulation stage are considered with respect to maximum conversion efficiency and output power. Section 5 reports on the methods used to conduct the experiments including simulations, a synthetic setup, a lab setup and a real-world setup. Section 6 comprises the experimental results including a characterization and comparison of two DC-DC conversion circuits and of two thermal harvesters for the wrist in a laboratory setup and in a real-world field test. In a case study we show how the optimized harvesters can be used to power a multi-sensor wearable, before we conclude in Section 7 .

\section{Related Work}

Over the last decade, wearable technology has received increasing attention from industrial as well as academic communities. Many commercial wearable devices became successful products in the wellness and sports domain and there is a number of applications that interface directly with a mobile phone [1. However, the main drawback reducing the success of wearable device is the limited battery lifetime. To overcome this limit, there are a number of approaches to 
capture energy from the environment, convert the input to a voltage range usable by connected electronics 13, 14 and store it in devices such as batteries or supercapacitors. As wearable devices are required to operate on the human body for long periods (i.e. days, or more), and cannot be supplied with energy by wires during use, achieving self-sustaining systems by energy harvesting is particularly attractive.

The most promising sources of energy for harvesting on and near the body include thermal [15, movement[7], light[16] and RF [8]. As humans frequently move, motion based harvesters are an obvious choice for wearable systems. The transducer can be piezoelectric [7, electromagnetic [17] or triboelectric [18] and are typically located on the limbs or integrated into a shoe. Power generation can be in the $\mu \mathrm{W}$ to $\mathrm{mW}$ range but is usually stated before conversion to usable voltages due to a number of challenges related to the power conversion. Body movements are irregular and low in frequency and do not allow for dynamic magnification using resonant designs. Finally in many usage scenarios where the wearer is stationary, no power can be generated at all. Most wearable energy harvesting systems today rely on photovoltaics (PV) as a main power source due to its convenience and high energy output in ideal operating conditions [16, 19]. PV cells can be flexible to increase comfort [20] and energy levels are in the lower $\mu \mathrm{W} / \mathrm{cm}^{2}$ range indoors to $\mathrm{mW} / \mathrm{cm}^{2}$ outdoors. In contrast to motion harvesters, voltage conversion with high efficiency is possible using commercial circuits. In 16 an ultra-low power multi-sensor wearable was equipped with a solar harvester and the authors demonstrated that the device can be self-sustainable for several days even in indoor conditions with a power consumption of $166 \mu \mathrm{W}$. Similarly to motion harvesters, PV power generation is highly situation specific and completely fails in many usage scenarios where insufficient light conditions are present. RF on the other hand is independent from light and movement but requires power lines or machinery in the direct vicinity and only provides a few nanowatts of harvested power [21, 8].

Thermoelectric energy conversion of human body heat represents a promising alternative as it is largely independent of external factors [22]. Lossec et al. used a theoretic approach to optimize the thermal system of TEG worn on the body [23. In [24] the authors reported on a wearable medical system that is powered by body heat and detects if a patient falls down. Leonov et al. presented a thermal harvester worn on the wrist that can be used to power a pulse oximeter [11. The authors demonstrated that in many indoor scenarios, the average power harvested per square centimeter is higher using the thermal harvester than a equally sized solar cell. However, the produced voltage is used to directly charge a supercapacitor as an energy buffer and the device is only operational if the ambient temperature is lower than $25^{\circ} \mathrm{C}$ to $27^{\circ} \mathrm{C}$. The same institute also applied TEG on the human forehead to power a 2-channel EEG system with a power consumption of $0.8 \mathrm{~mW}$ [25]. Up to $30 \mu \mathrm{W} \mathrm{cm} \mathrm{cm}^{-2}$ can be harvested before DC-DC conversion. A two-stage custom DC-DC converter design is used to convert the voltage produced by the TEG to $2.75 \mathrm{~V}$. Due to the large thermal harvester, the system has very limited wearability. Both previous systems rely on custom designed and fabricated components including the TEG 
and DC-DC circuitry to optimize the output power for a very specific application scenario. The authors further acknowledge that the high fabrication cost of their devices prevents widespread application and propose microfabricated TEG as a solution. To date however, commercial microstructured TEG are more expensive than conventional ones.

The presented approaches demonstrate that harvesting of human body heat is a valid alternative to other energy harvesting systems. However, application specific component manufacturing is necessary to obtain the power output required for a wearable sensor application. In [26, the authors use off-the-shelf components to harvest thermal energy from the wrist. The resulting power density at room temperature is $2.2 \mu \mathrm{W} \mathrm{cm}{ }^{-2}$, one order of magnitude lower than approaches using custom parts. Additionally, state-of-the-art thermal harvesters are typically too bulky and uncomfortable to achieve true wearability. Kim et al. presented a highly wearable harvester using TEG embedded in clothing but the acquired energy was only in the nanowatt range even at low ambient temperatures 27. Emerging flexible TEG that can adopt arbitrary shapes 9, 28, 29] promise a boost in wearability and power, but to date no harvesting in the higher microwatt range was demonstrated on the human body. An issue preventing further improvement of wearable thermal harvesters is that most approaches focus either solely on the thermal component [30, 31, 26], the DCDC conversion circuit [32, 33, 34] or on the low-power electronics [35, 36, 37, resulting in a mismatch of the individual stages and therefore waste of usable power.

In contrast, we propose a complete system optimization from the energy source (the human body), over the power conversion circuitry to the hardware utilized in a specific application to achieve maximum output power and wearability. The optimization includes a simulation of the complete harvester, a laboratory characterization of individual and assembled components and an evaluation of the system in the field. We put special emphasis on the critical interaction between thermal harvester and DC-DC conversion stage and use low-cost, off-the-shelf components to demonstrate that thermal harvesting can supply a wearable hardware platform in real-life situations.

\section{Thermoelectric Energy Conversion}

In this section we focus on the thermal and thermoelectric generator part of the pathway from human body heat to application shown in Figure 1.

\subsection{Thermoelectric Power Generation and Conversion Efficiency}

To exploit the thermoelectric effect for power generation, two thermolegs with different Seebeck coefficients $\left(\alpha_{m 1}\right.$ and $\left.\alpha_{m 2}\right)$ are combined to form a thermocouple (TC). A number $m$ of thermocouples that are connected electrically in series and thermally in parallel form a TEG. The application of a temperature 
difference $\Delta T_{T E G}=T_{\text {hot }}-T_{\text {cold }}$ to the TEG results in an open circuit voltage $V_{O C}$ according to:

$$
V_{O C}=\alpha \cdot \Delta T_{T E G}
$$

where $\alpha=\alpha_{m 1}-\alpha_{m 2}$ is the combined Seebeck coefficient of the TC. The maximum output power $P_{\max }$ for an electrically matched load and for small $\Delta T_{T E G}$ can then be approximated as [38]:

$$
P_{\text {max }}=\frac{V_{O C}^{2}}{4 \cdot R_{e l}}=\frac{(m \cdot \alpha)^{2}}{4 \cdot R_{e l}} \cdot\left(\Delta T_{T E G}\right)^{2},
$$

where $R_{e l}$ is the internal electrical resistance of the TEG. To quantify the ability of a TEG for thermoelectric power conversion, a module figure of merit $Z T_{m}$ can be defined similar to the thermoelectric figure of merit $Z T$ [39] as

$$
Z T_{m}=\frac{(m \cdot \alpha)^{2} K_{T E G}}{R_{e l}},
$$

where $K_{T E G}$ is the thermal resistance of the generator. If an ideal Carnot engine is connected via thermal interface resistances to the heat reservoir (e.g. the human body, $\left.T_{b o d y}\right)$ and the heat sink (e.g. the ambient air, $T_{a m b}$ ), the maximum conversion efficiency is calculated as 40.

$$
\eta_{\text {ideal }}=1-\sqrt{\frac{T_{a m b}}{T_{b o d y}}}
$$

for an electric and thermally matched generator. Taking parasitic losses by thermal conduction and joule heating into account, the conversion efficiency of a system optimized for power output is reduced to

$$
\eta_{\text {real }}=\eta_{\text {ideal }} \frac{Z T}{(1+\sqrt{1+Z T})^{2}} .
$$

The correction term on the right side is defined by the material properties of the TEG [41. Equation (5) shows that, prior to DC-DC conversion, the maximum output power of a fully matched system is only dependent on the ZT of the thermoelectric material whereas higher ZT results in higher power. To obtain the total conversion efficiency $\eta_{\text {total }}$ from body core heat to an application platform, thermal and electric conversion efficiency has to be combined to

$$
\eta_{\text {total }}=\eta_{\text {real }} \cdot \eta_{e l} .
$$

The electric conversion efficiency $\eta_{e l}$ will be discussed in the following section. 
Table 1: Typical characteristics of $\mu$ TEG and mTEG

\begin{tabular}{l|cc} 
Characteristic & pTEG & mTEG \\
\hline TC Number/Density & high $\left(\geq 100 \mathrm{~cm}^{-2}\right)$ & low $\left(<100 \mathrm{~cm}^{-2}\right)$ \\
Open Circuit Voltage & high $\left(\geq 50 \mathrm{mV} \mathrm{K}^{-1} \mathrm{~cm}^{-2}\right)$ & low $\left(<50 \mathrm{mV} \mathrm{K}^{-1} \mathrm{~cm}^{-2}\right)$ \\
Electric Resistance & high $\left(\geq 50 \Omega \mathrm{cm}^{-2}\right)$ & low $\left(<50 \Omega \mathrm{cm}^{-2}\right)$ \\
Thermal Resistance & low $\left(<15 \mathrm{~cm}^{2} \mathrm{~K} \mathrm{~W}^{-1}\right)$ & high $\left(\geq 15 \mathrm{~cm}^{2} \mathrm{~K} \mathrm{~W}^{-1}\right)$ \\
Thermoleg Cross Section & small $\left(<0.2 \mathrm{~mm}^{2}\right)$ & large $\left(\geq 0.2 \mathrm{~mm}^{2}\right)$ \\
Thermoleg Length & short $(<900 \mu \mathrm{m})$ & long $(\geq 900 \mu \mathrm{m})$ \\
\hline
\end{tabular}

\subsection{TEG Classification}

TEG can be generally classified in mTEG that have macroscopic thermolegs and are manufactured using classic fabrication technology (e.g. Quickohm, Thermalforce), and $\mu$ TEG that have a high number and density of TC and are produced with microfabrication techniques (e.g. greenTEG, Micropelt). In Table 1, we use existing classification markers from literature, namely thermoleg length and cross section [42, Chapter 12], and identify further typical attributes including TC density, normalized open circuit voltage and normalized electric and thermal resistance. Besides their small size and weight, the high number of TC in $\mu$ TEG results in high open circuit voltages at comparably small temperature differences. This makes them seemingly ideal candidates for wearable applications. However, their $Z T_{m}$ is typically low compared to mTEG due to low thermal but high electric resistances. Additionally, to avoid parasitic heat losses, the thin $\mu$ TEG are commonly packaged between thermally conductive spacers revoking their size and weight advantage. In this work we compare the suitability of both approaches for harvesting of body heat by using a state-of-theart representative of each class: A high-output voltage, high electric resistance pTEG (Micropelt TPG-651) versus a high thermal but low electric resistance mTEG (Quick-cool QC32-0.6-1.2). Both TEG fulfill the specifications of their respective category listed in Table 1 .

\subsection{Thermal Harvesters for Human Body Heat}

The human body can be represented by a thermal resistance circuit as shown in Figure 1-Thermal. A temperature difference $\Delta T=T_{b o d y}-T_{a m b}$ between the body core and the environment is the driving force of a heat flux from the human body, through the harvester into the environment. $K_{b o d y}, K_{T E G}$ and $K_{\text {sink }}$ are the respective thermal resistances whereas interface resistances are included in $K_{\text {body }}$ and $K_{\text {sink }}$. The two presented TEG types are combined with thermal interfaces to the skin and to the ambient air in order to use them as thermal harvesters on the human body as shown in Figure 2a.

Each element of a harvester module can be optimized for a given application: (1) Thermal interfaces between skin and TEG increase the effective harvesting area of the system and funnel acquired heat to the generator. Because the thermal contact resistance to the skin is inversely proportional to the contact area, larger interfaces allow for better thermal matching of TEG and interfaces. In this study, we use aluminum plates $(13 \times 20 \times 2 \mathrm{~mm})$ as hot interface for both 


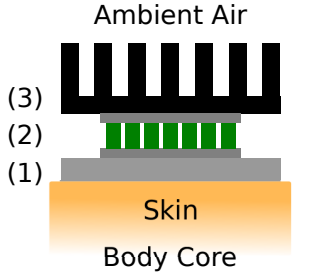

(a) Thermal harvester module (b)

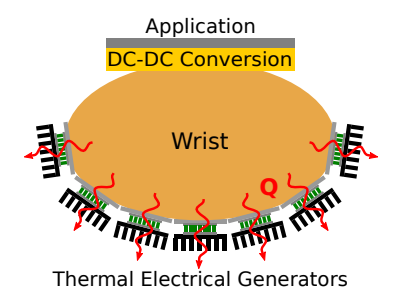

b) TEG arrangement on wrist

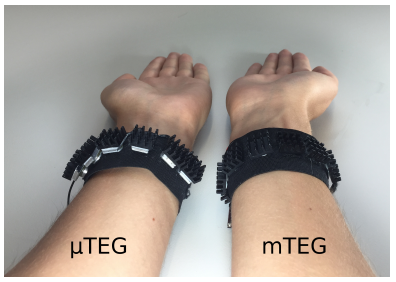

(c) Wristband prototype

Figure 2: (a) Schematic of a thermal harvester module on the human body: (1) Thermal interface to the heat source/skin, (2) TEG for thermal to electric power conversion, (3) Heat sink dissipating heat into the ambient air. (b) Schematic of body-heat powered wearable for the wrist including energy harvester, DC-DC conversion and storage and application circuit. Seven harvesting modules are connected electrically in series and thermally in parallel. (c) Picture of worn harvesting wristbands: mTEG approach (left) and $\mu$ TEG approach (right).

TEG approaches due to their light weight and high thermal conductivity.

(2) To obtain maximum output power, the electric resistance of the TEG should be matched with the electric load $R_{\text {el,ideal }}=R_{\text {load }}$, and the thermal resistance with the combined resistances of the heat source and the environment $K_{T E G, \text { ideal }}=K_{\text {body }}+K_{\text {sink }}$ [3]. Thermal and electric resistance are directly coupled over the number of thermolegs $m$ and their ratio of length to crosssectional area $l / A$. Assuming that the thermal conductivity of the filler material between TC (e.g. air) is small compared to the thermoelectric material $\lambda_{\text {fill }} \ll \lambda_{n, p}$ and that the Peltier effect can be neglected, the ideal number of TC $m_{\text {ideal }}$ to achieve both thermal and electric matching can be calculated from the ratio of $R_{\text {el,ideal }} / K_{T E G, \text { ideal }}$ 44]:

$$
m_{\text {ideal }}=\sqrt{\frac{R_{\text {load }}}{\left(K_{\text {body }}+K_{\text {sink }}\right)} \frac{\left(\sigma_{n}+\sigma_{p}\right)}{\left(\lambda_{n}+\lambda_{p}\right)}},
$$

where $\sigma_{n}$ and $\sigma_{p}$ is the electric and $\lambda_{n}$ and $\lambda_{p}$ the thermal conductivity of the n- and p-doped thermolegs and where all TC are connected electrically in series and thermally in parallel. The ideal ratio of thermoleg length to area $(l / A)_{\text {ideal }}$ can then be calculated:

$$
\begin{aligned}
(l / A)_{\text {ideal }}=\frac{R_{\text {load }}}{m_{\text {ideal }}}\left(\sigma_{n}+\sigma_{p}\right) & = \\
& \sqrt{R_{\text {load }}\left(K_{\text {body }}+K_{\text {sink }}\right)\left(\lambda_{n}+\lambda_{p}\right)\left(\sigma_{n}+\sigma_{p}\right)}
\end{aligned}
$$

(3) Heat sinks dissipate heat traversing the thermal harvester into the ambient air. Larger heat sinks (or heat sinks with lower thermal resistance) result in larger output power if thermal matching is maintained. For a wearable application however, their weight and size needs to be minimized. Fin- or pin-type aluminum heat sinks are commonly used due to the small thermal resistance per volume. A problem for human body applications is unpleasant cooling of the 
Table 2: Specifications of harvesters used in this study.

\begin{tabular}{l|cc} 
Specification & pTEG Approach & mTEG Approach \\
\hline Area $\left[\mathrm{cm}^{2}\right]$ & 20 & 20 \\
Height $[\mathrm{mm}]$ & 17 & 11 \\
Weight $[\mathrm{g}]$ & 39 & 41 \\
Thermal Resistance $\left[\mathrm{K} \mathrm{W}^{-1}\right]$ & $7 \times 28$ & $7 \times 42$ \\
Electric Resistance $[\Omega]$ & 1470 & 20 \\
Module Seebeck Coefficient $\left[\mathrm{V} \mathrm{K}^{-1}\right]$ & 0.42 & 0.095 \\
Module ZT [-] & 0.15 & 0.83 \\
DC-DC Conversion Circuit & bq25504 & LTC3108 \\
\hline
\end{tabular}

skin for large sinks in low ambient temperatures [25. Accordingly, the choice of the heat sink needs to be matched with the ambient temperature range of the application scenario. In this study we use commercial heat sinks (Fischer, $14 \times 14 \times 6 \mathrm{~mm}, K_{\text {sink }}=29 \mathrm{~K} \mathrm{~W}^{-1}$ ) for both TEG approaches. For all three components of the harvester module, a compromise between output power and wearability needs to be achieved.

\subsection{Thermal Harvesting Systems Evaluated in this Study}

A schematic for the body heat powered wearable used in this study is shown in Figure 2b. Seven harvester modules can be attached to the wrist while maintaining high wearability and are connected electrically in series and thermally in parallel to maximize the produced open circuit voltage. The thermal harvester is connected to a DC-DC conversion and energy storage circuit, an application circuit completes the device. All elements integrate into an elastic band that wraps comfortably around the wrist. An image of two assembled devices, one based on $\mu$ TEG (left) and one on mTEG (right) is shown in Figure 2c. The specifications of both harvesters are listed in Table 2. The table highlights the fundamental differences of both approaches regarding thermal and electric resistances as well as module Seebeck coefficient and figure of merit. Although both harvesters have a similar material $Z T$, the $Z T_{m}$ of the $\mu$ TEG is significantly lower compared to the mTEG. The four times higher module Seebeck coefficient $m \alpha$ of the $\mu$ TEG is compensated by the high electric resistances of the microscopic thermolegs and interconnects. This indicates a higher output power of the mTEG prior to DC-DC conversion. Using (7) and (8) and typical material parameters for thermocouples and thermal interfaces, we can compare how close the two used TEG come to ideal electric and thermal matching conditions. The $\mu$ TEG has a total of $m_{\mu, \text { real }}=1622 \mathrm{TC}\left(m_{\mu, \text { ideal }}=1051 \mathrm{TC}\right)$ and the mTEG of $m_{m, \text { real }}=224 \mathrm{TC}\left(m_{m, \text { ideal }}=123 \mathrm{TC}\right)$. The increased number of TC in the real system ( $\mu$ TEG: $+54 \%$, mTEG: $+84 \%$ ) is required to satisfy the minimum input voltage requirements of DC-DC converters and shows that optimization can not solely be based on thermal aspects. Using (8), we find that only about $10 \%$ of the ideal TC length-to-area ratio is achieved for the pTEG and $31 \%$ for the mTEG. The main limitations preventing higher aspect 
Table 3: Comparison of two commercial state-of-the-art DC-DC converter architectures for TEG harvesting.

\begin{tabular}{l|cc} 
Parameter & Single Inductor & Coupled Inductors \\
\hline Example Circuit & bq25504 [45] & LTC3108 [46] \\
Switching Control & active feedback control & passively controlled switch \\
Startup Voltage & $330 \mathrm{mV}$ & down to $20 \mathrm{mV}$ \\
Min. Input Voltage & $\geq 100 \mathrm{mV}$ & down to $20 \mathrm{mV}$ \\
Efficiency & up to $85 \%$ & up to $50 \%$ \\
Voltage Regulation & controlled boost ratio & linear drop-out \\
Leakage & boost control circuit & passive switching, rectification, \\
& & inductor coupling \\
\hline
\end{tabular}

ratio is the microfabrication process of the $\mathrm{TTEG}$ and the mechanical stability in the mTEG.

The choice of the DC-DC conversion circuits and the importance of matching them with the complete system will be discussed in the following section.

\section{DC-DC Conversion and Voltage Regulation}

The low temperature differences and the small sized TEG modules for wearability result in low output voltages and power of the transducers. Therefore, it is inevitable to convert the voltage from at maximum a few hundred millivolts up to several volts to charge a battery or supercapacitor and supply the application circuit (Figure 1- Voltage Regulation). We consider state-of-theart DC-DC converters that are designed for voltage conversion in generic TEG applications. We compare the two typically used architectures using a representative, commercially available harvesting circuit of each category. Because an efficient supply of the application circuit is also of high importance for the overall system efficiency $\eta_{\text {total }}$, the selection of the voltage regulator among different available options is also being discussed.

\subsection{DC-DC Conversion Trade-Offs}

The architectures of state-of-the-art DC-DC converters can be divided in two broad categories: actively controlled single inductors and passively switched coupled inductors without any control circuit. A summary of how these two architectures compare is given in Table 3 . The differing internal DC-DC conversion architectures and the necessary external circuitry are shown in Figures 3 and 4 . For the following discussion of the trade-offs when deploying these architectures in a wearable harvesting scenario, we take two specific circuits as example. As representative examples, the bq25504 from Texas Instruments [45] for a single inductor based DC-DC converter, and the LTC3108 from Linear Technology [46] for a coupled inductors based solution were selected.

The advantage of single inductor based solutions is their ability to match the input impedance and boost-up ratio dynamically depending on the harvesting situation using its internal control circuit. This allows maximum power point 


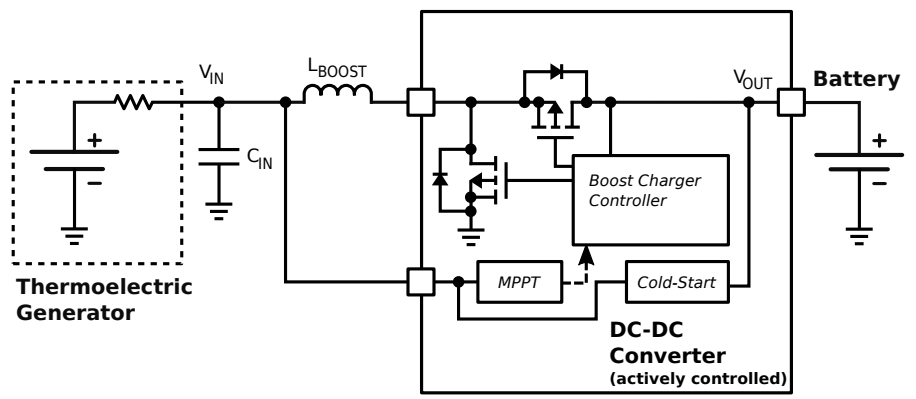

Figure 3: Single inductor based DC-DC conversion architecture (adapted from [45]).

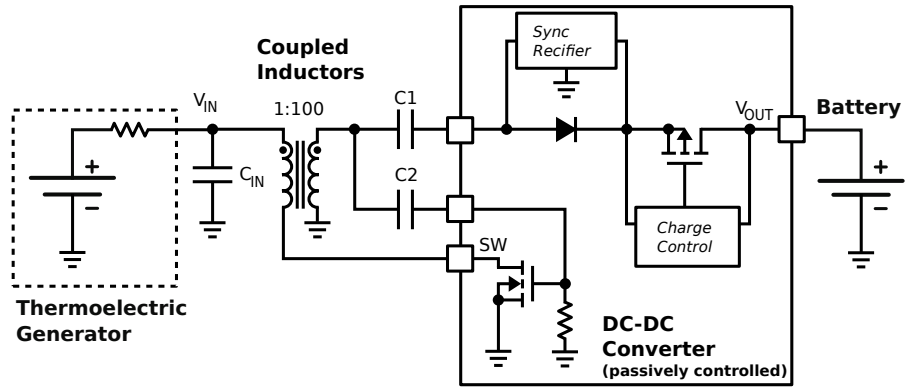

Figure 4: Coupled inductors based DC-DC conversion architecture (adapted from [46]).

tracking (MPPT) and guarantees efficient conversion also under changing harvesting conditions. This results in high efficiency of $60 \%$ even for low input voltages and increases beyond $90 \%$ for higher input voltages and power. However, the flexibility of this architecture comes with a limited boost-up ratio, and consequently a comparably high minimum input voltages of $100 \mathrm{mV}$ and more. Additionally, the unit controlling the inductor switching and adaption of the input impedance requires a voltage supply for the converter to work. Therefore, an additional passive cold-start voltage converter with several hundred millivolts start-up voltage and low efficiency is required to charge to the initial voltage level where the control circuit and high efficiency harvesting start operating.

The advantage of coupled inductors based DC-DC converters is a very small input startup voltage of only $20 \mathrm{mV}$. Due to the fully passive circuit design, no separate startup converter is needed to start operation. However, the passive switching circuit, inductor coupling and rectification required after the boosting stage come with their own inefficiencies, resulting in a reduced conversion efficiency. Additionally, the fixed boost ratio defined by the inductors results in a boosted voltage that gets much higher than the battery voltage for higher input voltages. Therefore, a passively controlled low-dropout (LDO) regulator is used internally for battery charge management. These effects result in peak efficiency of $50 \%$ for voltages in the region above the startup voltage. For higher input voltages, however, the efficiency decreases linearly due to the inter- 
Table 4: Comparison of two typical voltage regulation architectures.

\begin{tabular}{l|cc} 
Parameter & Low-Dropout Regulator & Buck Converter \\
\hline $\begin{array}{l}\text { Typical Use-Case } \\
\text { Voltage Regulation } \\
\text { Losses }\end{array}$ & $\begin{array}{c}\text { low-currents } \ll 1 \mu \mathrm{A} \\
\text { resistance regulation } \\
\text { input/output voltage } \\
\text { difference }\end{array}$ & $\begin{array}{c}\text { high voltage difference } \\
\text { switching control circuit } \\
\text { control circuit, } \\
\text { inductor switching }\end{array}$ \\
\hline
\end{tabular}

nal LDO down regulation. Furthermore, the fixed input impedance a few ohms restricts the range of compatible TEG modules to a subset of mTEG in order to achieve optimal impedance matching.

Voltages of $120 \mathrm{mV}$ and more produced by $\mathrm{TTEG}$ presented in Section 3.4 guarantee operation with the bq25504, an actively controlled single inductor DC-DC converter. This converter handles the increased internal resistance of $\mu$ TEG due to flexible impedance matching using MPPT. The fixed, low input impedance of coupled inductors based circuits like the LTC3108 does not allow harvesting with the high internal resistance of $\mu \mathrm{TEG}$, as will be confirmed in Section 6.1. However, the low startup voltage of the LTC3108 allows harvesting with the mTEG wristband, even though this TEG wristband has a very low output voltage of $\leq 50 \mathrm{mV}$ at room temperature. Also, the low resistance of mTEG allows combining multiple modules while still guaranteeing sufficient impedance matching. A bq25504 will not work in combination with mTEG in the considered wearable scenario, because of the very low input voltage.

\subsection{Application Supply}

An intermediate energy buffer such as a battery or supercapacitor and a separate voltage regulation for the application hardware guarantees full decoupling of the harvesting and application voltages. This allows for both harvester and load to work at their individual optimal operation point for optimal energy efficiency [47.

For the overall system efficiency also the output voltage regulators need to be chosen carefully. A comparison of typically used low-dropout and buck regulators is shown in Table 4 . While buck converters feature a very high efficiency of $>94 \%$ at active currents of $1 \mathrm{~mA}$ and more, they require power consuming inductor switching and control also at sleep currents in the nA range. Low-dropout (LDO) regulators on the other hand have a simple circuit with minimal overhead to regulate their series resistance in the voltage supply. This results in a low efficiency compared to buck converters if the application voltage needs to be reduced significantly, but performs much better in the ultra-low current region of $\ll 1 \mu \mathrm{A}$. 
The optimal output regulator needs to be adjusted to the application's hardware platform: if the system uses very aggressive duty-cycling an LDO might be advantageous, because it handles the sleep state of the hardware more efficiently. A buck converter performs better when the application hardware's supply voltage is much lower than the battery voltage due to higher efficiency during active periods.

With this we completed the discussion of the trade-offs and design parameters of the individual sub-components illustrated in Figure 1. In a next step we discuss the experimental setups that were used to evaluate the discussed harvesting solutions.

\section{Simulation and Experimental Setup}

In this section the simulation and experimental setups are introduced. As a first step we simulate the performance of the used thermal harvesting systems and characterize the DC-DC converter efficiency. We then evaluate the assembled harvesting systems, first in a controlled lab setup and later in real-world experiment.

\subsection{Simulation}

We use a Matlab script to simulate the output characteristics of different thermal harvesting systems for distinct body locations and in varying environmental conditions. The simulation is based on a 1-D thermal resistance model as proposed in literature 12 and shown in Figure 1 1 - Thermal. Equivalent to an electrical circuit, the potential is represented by the temperature difference $\Delta T$, the current by the heat flux $Q$, and the electrical resistances by thermal resistances $K$. We first compute the temperature difference across the TEG $\left(\Delta T_{T E G}\right)$ by solving the equations for the incoming and outgoing heat flux through the TEG $\left(Q_{\text {in,TEG }}, Q_{\text {out }, T E G}\right)$ after $\Delta T_{T E G}=T_{\text {hot }}-T_{\text {cold }}$ [38]. Based on the individual characteristics, stacking and wiring of the TEG, the open circuit voltage and the output power for dynamic electrical load matching and before DC-DC conversion is then calculated using (2). We extend the existing model with the voltage and resistance dependent conversion efficiency $\eta_{e l}=f\left(V_{i n}, R_{i n}\right)$ of the DC-DC converter discussed below to simulate the output power supplied to a rechargeable battery at $3.7 \mathrm{~V}$ :

$$
P_{\text {out }, 3.7 \mathrm{~V}}=P_{\max } \cdot \eta_{\text {el }}
$$

\subsection{DC-DC Converter Characterization Setup}

In this experiment we characterize the efficiency of two commercial DCDC conversion circuits for thermal energy harvesting. The efficiency for the bq25504 from Texas Instruments and the LTC3108 from Linear Technology are evaluated for a wide range of internal resistances and open circuit voltages of the source. The TEG module is emulated with the equivalent circuit model using a Keithley 2220-30-1 power supply with constant voltage $V_{O C}$ connected in series 
with a resistance of $R_{e l}$. This allows the evaluation of a wide range of TEG properties and harvesting conditions. At the output of the harvesting circuits a source meter (Keithley SMU 2450) is used to emulate a typical Lithium-Polymer battery at $3.7 \mathrm{~V}$, while measuring the harvested power at the same time. The resulting harvesting power $P_{\text {harv }}$ is then calculated from the extracted current at the output of the harvesting circuit. Comparing this value with the maximum power given in (2), the electrical harvesting efficiency is finally calculated as:

$$
\eta_{e l}=\frac{P_{h a r v}}{P_{\max }}
$$

\subsection{Laboratory Test Setup}

The assembled harvesting systems summarized in Table 2 are tested and characterized under controlled lab conditions. The setup is based on a temperature regulated water bath on the hot side, and an air conditioned lab room that represents the colder ambient side. The water bath temperature is increased linearly over several hours to evaluate the harvesting system for different temperature differences $\Delta T$ and $\Delta T_{T E G}$. The harvester wristbands are mounted symmetrically one on each side of the water bath to guarantee the same operating conditions. We use T-type thermocouples (Labfacility Z2-T-1) placed directly at the hot and cold surface of one TEG in each wristband to measure the actual temperature difference across the TEG terminals $\Delta T_{T E G}$ with an Omega OM-DAQPRO data logger. All TEG of each wristband are connected in series to the input of their corresponding DC-DC converter. Analogous to the harvester characterization, a source meter is used to measure the extracted power and to emulate the nominal voltage of $3.7 \mathrm{~V}$ of Lithium-Polymer batteries. In an actual system integration, any Li-Ion battery or supercapacitor can be used without additional protection circuitry, as both harvesting circuits feature battery overcharge protection. By logging the current the SMU extracts from the harvesting circuit at a constant voltage, the total harvested power can be calculated. Combining these measurements with the recorded temperature difference $\Delta T_{T E G}$, the harvested power for different temperatures can be analyzed.

\subsection{Real-World Test Setup}

The laboratory setup described in Section 5.3 is extended by a portable power supply and integrated into a backpack to allow mobile real-world measurements on the human body. Instead of a water bath, the human body serves as the heat source. The mTEG and $\mu$ TEG wristbands are strapped to the left and right wrist of the test subject at the same position and with the same attachment pressure. A second person carries the backpack containing the measurement devices to allow for free and natural movement of the test subject. The test subject then performs a set of predefined activities like sitting still and walking around in environments with different ambient temperatures. Dividing the power harvested in the real-world experiment $P_{\text {harv }}$ by the power 


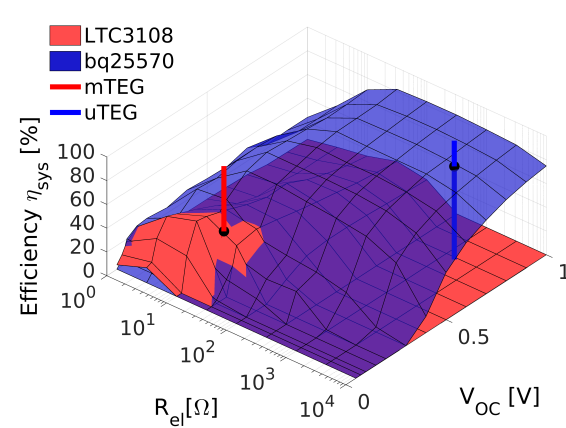

(a) DC-DC converter efficiency compared to the maximum power point 10 .

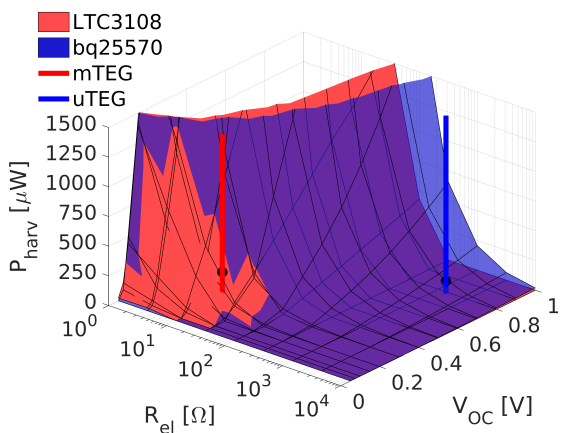

(b) Absolute harvested power Pharv transferred to the storage.

Figure 5: Harvesting circuit evaluation results for wide range of emulated TEG with internal resistance $R_{e l}$ and open circuit voltage $V_{O C}$.

$P_{b o d y}=Q_{b o d y} \cdot A_{\text {harv }}$ leaving the human body, the measured total energy conversion efficiency is calculated as

$$
\eta_{\text {meas }}=\frac{P_{h a r v}}{Q_{\text {body }} A_{\text {harv }}},
$$

where $Q_{\text {body }}$ is the heat flux leaving the body and $A_{\text {harv }}$ is the area of the harvester's interface.

\section{Results and Discussion}

This section presents the experimental results together with the discussion of the observed system behavior. A case study with an ultra-low power mulitsensor bracelet will show that thermoelectric energy harvesting provides sufficient energy for real-world applications.

\subsection{DC-DC Converter Characterization}

The DC-DC characterization results for internal resistances ranging from $1 \Omega$ to $10 \mathrm{k} \Omega$ and open circuit voltages of $0.05 \mathrm{~V}$ to $1.0 \mathrm{~V}$ are shown in Figure 5 . Figure 5 a represents the overall electric conversion efficiency $\eta_{e l}$ which was defined in (10), and Figure $5 \mathrm{~b}$ shows the absolute harvested power $P_{\text {harv }}$.

The LTC3108 DC-DC converter features high efficiency at low voltages $V_{O C}$ and low to medium internal resistances $R_{e l}$, the operating region for which its input impedance and boost ratio are specifically designed. However, the efficiency decreases for higher voltages and resistances. For input impedances of $\geq 316 \Omega$ no energy can be harvested, because of the mismatch of the converter's fixed low input impedance with the generator. For high input voltages $V_{O C}$ and medium to high internal resistances $R_{e l}$, the bq25504 can adapt its input impedance, resulting in high harvesting efficiency. The peak efficiencies of the 


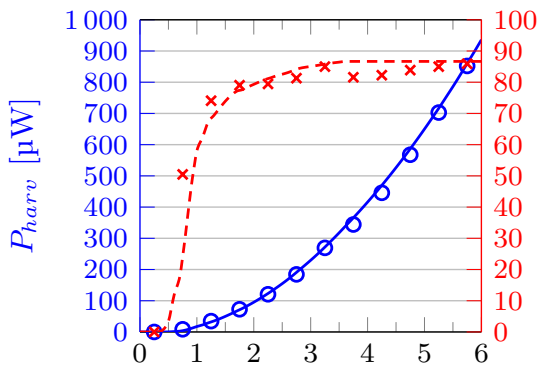

Temperature Difference $\Delta T_{T E G}[\mathrm{~K}]$

(a) bq25504 and 7 TPG-651 $\mu$ TEG.

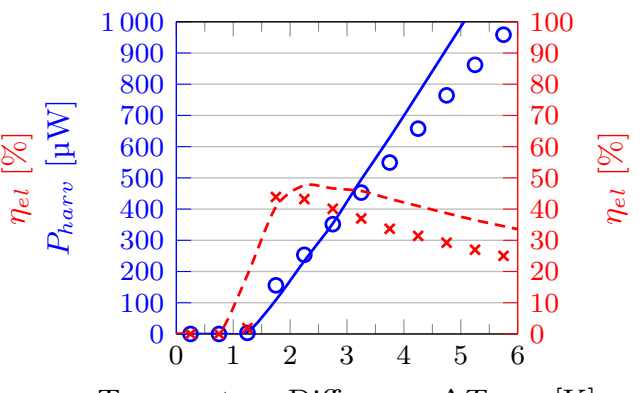

Temperature Difference $\Delta T_{T E G}[\mathrm{~K}]$

(b) LTC3108 and 7 QC-32-0.6-1.2 mTEG.

Figure 6: Harvested power $P_{\text {harv }}$ and the electric harvesting efficiency $\eta_{e l}$ dependent on the temperature difference $\Delta T_{T E G}$ between the TEG terminals for two harvesting systems. The plots show simulations of the DC-DC efficiency (dashed lines) and the harvested power (solid lines) for both $\mu$ TEG and mTEG approach. The real conversion efficiency (crosses) is derived from the measured power (circular markers) using 10 .

DC-DC converters reach their maximum of $52.5 \%$ at $10 \Omega$ and $0.15 \mathrm{~V}$ for the LTC 3108 , and $83.3 \%$ at $316 \Omega$ and $1.0 \mathrm{~V}$ for the bq25504. The plots also show the expected operating points at room temperature for the used mTEG and $\mu$ TEG harvesting systems discussed in Section 3.4. The mTEG performs better in combination with the LTC3108, while the $\mu$ TEG only works with the bq25504 and with a very high electric conversion efficiency. Perfect thermal matching of the real TEG would move the operation points slightly along the $V_{O C}$ axis but does not change the electrical resistance. Only improvements of the figure of merit $(Z T)$ could shift both harvesters into the higher power regime by providing high voltages at lower electric resistance.

\subsection{Lab Evaluation of the Harvesting System}

The analysis of the harvesting power $P_{h a r v}$ and efficency $\eta_{e l}$ as a function of the temperature difference $\Delta T_{T E G}$ is shown in Figures $6 \mathrm{~b}$ and $6 \mathrm{a}$. The generated power $P_{\text {harv }}$ was first simulated and then verified in the laboratory setup discussed in Section 5.3. The electrical harvesting efficiency $\eta_{e l}$ represents the harvested power compared to the theoretical maximum as defined in (10).

A minimum temperature difference of $1 \mathrm{~K}$ is required for the bq25504 DCDC conversion circuit and the seven $\mu$ TEG wristband to harvest energy. The high minimal voltage is already reached at low differences due to the high output voltage of the $\mu$ TEG. At the same temperature difference the efficiency increases step function like, reaching a maximum of $85.9 \%$ at $5.75 \mathrm{~K}$ difference. Being able to adapt to varying harvesting conditions, the bq25504 does not show any decrease in efficiency once the minimal operation conditions are reached. This results in a quadratic increase of the harvesting power with the temperature difference from $8.5 \mu \mathrm{W}$ at $0.75 \mathrm{~K}$ to $852.1 \mu \mathrm{W}$ at $5.75 \mathrm{~K}$.

With seven mTEG and the LTC3108 DC-DC conversion circuit harvesting starts at a minimum temperature difference of $1.25 \mathrm{~K}$. Before transferring 
energy to the battery, the minimal startup voltage and the comparably high selfconsumption of $24.8 \mu \mathrm{W}$ needs to be provided. The peak efficiency of $43.9 \%$ is reached at $1.75 \mathrm{~K}$ and then decreases linearly due to the fixed boost ratio and the internal down regulation of the boosted voltage (discussed in Section 4.1). As a result, the harvested power increases only linear with the temperature difference. However, ranging form $3.4 \mu \mathrm{W}$ at $1.25 \mathrm{~K}$ to $958.6 \mu \mathrm{W}$ at $5.75 \mathrm{~K}$ temperature difference, the power harvested is still significant.

Applying the module figure of merit $Z T_{m}$ of both approaches (see Table 2 ) in (5) results in approximately four times the thermoelectric conversion efficiency for the mTEG compared to the $\mu$ TEG $\left(\eta_{\text {real }, m} \approx 4 \cdot \eta_{\text {real }, \mu}\right)$. Combined with the electric conversion, approximately $65 \%$ higher total output power can be expected for the mTEG in temperature differences $\Delta T_{T E G}>1.75 \mathrm{~K}$ confirming the experimental results. These results are remarkable considering that the module $Z T_{m}$ of the mTEG is more than five times larger than the $Z T_{m}$ of the $\mu$ TEG and once more demonstrate the importance of a complete system optimization. Comparing the lab experiment to the simulation, we find that we can accurately predict the harvested power supplied to a battery at $3.7 \mathrm{~V}$ and in low temperature differences as they occur in human body applications. The average error is $4.6 \mu \mathrm{W}(2.1 \%)$ over the complete measured temperature range for the $\mu$ TEG approach and $44.0 \mu \mathrm{W}(15.8 \%)$ for the mTEG assembly. While the $\mu$ TEG power is accurate from $0 \mathrm{~K}$ to $6 \mathrm{~K} \Delta T_{T E G}$ the mTEG output deviates for temperature differences larger $3 \mathrm{~K}$ due to a mismatch in simulated and measured conversion efficiency. This can be a result of the limited number of measurement points acquired in Section 6.1 and the lack of maximum power point tracking in the LTC3108.

\subsection{Real-World Evaluation of Harvester Circuits}

Figure 7 shows the real-world evaluation of the two harvesting approaches worn on the human wrist. The harvesting power is evaluated in three ambient temperature conditions: $23^{\circ} \mathrm{C}$ in an office building, $12{ }^{\circ} \mathrm{C}$ to $15{ }^{\circ} \mathrm{C}$ outdoors, and $18^{\circ} \mathrm{C}$ in an underground parking lot. For each environmental condition the subject was first stationary (standing or sitting) and then walking at moderate speed. Walking along corridors inside the building results in an initial power of $230 \mu \mathrm{W}$ before the power reduces to $90 \mu \mathrm{W}$ for thermal equilibrium. Leaving the building and sitting outdoors, the harvesting power is on average $370 \mu \mathrm{W}$ for the $\mu$ TEG and $390 \mu \mathrm{W}$ for the mTEG approach. While walking outdoors, the power generation reaches $750 \mu \mathrm{W}$ to $1080 \mu \mathrm{W}$ for both approaches. Larger fluctuations in outdoor measurements originate from gusts of wind. Entering the building with cooled skin from outdoors, the temperature difference across the TEG collapses and the LTC3108 temporarily stops harvesting completely. In the parking, both harvesters produce around $70 \mu \mathrm{W}$ of power while sitting and between $150 \mu \mathrm{W}$ and $200 \mu \mathrm{W}$ while walking. In the worst-case scenario, the test subject is sitting in an office at $23^{\circ} \mathrm{C}$. Even without any movement or air convection, $50 \mu \mathrm{W}$ can be harvested on average with both harvesting systems.

During the overall measurement, an average power of $280 \mu \mathrm{W}\left(14 \mu \mathrm{W} \mathrm{cm}{ }^{-2}\right)$ for the mTEG and of $260 \mu \mathrm{W}\left(13 \mu \mathrm{W} \mathrm{cm}{ }^{-2}\right)$ for the $\mu$ TEG approach was har- 


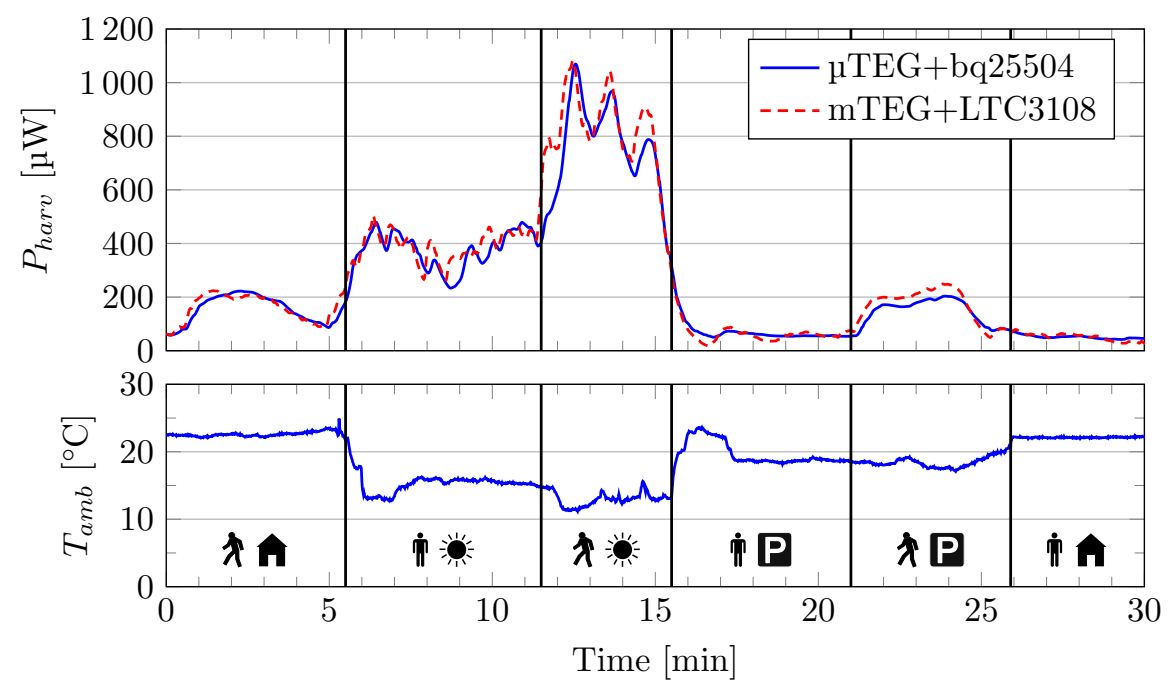

Figure 7: Evaluation results of the harvesting power $P_{h a r v}$ for both thermal harvesting systems for the real-world experiments with ambient temperature $T_{a m b}$.

Scenario legend: $\mathbf{A}$ indoor, outdoor, $\mathbf{P}$ underground parking, $\dot{\prod}$ standing/sitting, $\boldsymbol{\Uparrow}$ walking.

vested. At low temperature differences of $\Delta T_{T E G}<1.75^{\circ} \mathrm{C}$ as they occur indoors, both solutions perform nearly identical. For larger differences up to $6{ }^{\circ} \mathrm{C}$ for an active subject outdoors, the superior module $Z T_{m}$ of the mTEG compensated for the lower electric conversion efficiency of the LTC3108. The resulting output power is $4 \%$ to $15 \%$ higher but does not reach the expected value from the laboratory evaluation. A likely reason is the active regulation of heat flux leaving the human body. Using vasoconstriction, the body tries to maintain a constant temperature and therefore compensates for differences in the heat flow inherent in the two harvester approaches. Different placement position, attachment pressure and uneven air convection are further possible sources of error. Assuming that the real-world evaluation is extended to a working day of 8 hours, $8.64 \mathrm{~J}$ of energy could be accumulated, enabling a number of interesting low-power applications.

Even in low temperatures outdoors, none of the test subjects noticed an uncomfortable feeling of cold due to the harvesters. The usable temperature of $10^{\circ} \mathrm{C}$ to $37^{\circ} \mathrm{C}$ is therefore significantly increased compared to state-of-theart systems. The test subjects also reported a unobtrusive user experience comparable to a watch.

\subsection{Total Efficiency of Energy Conversion}

Approximately $10 \mathrm{~mW}$ to $100 \mathrm{~mW}$ of heat per square centimeter leave the human skin depending on body location, environmental conditions and activity of the wearer [48. On the wrist the heat flux $Q_{b o d y}$ varies between $18 \mathrm{~mW} \mathrm{~cm}^{-2}$ for a stationary and $80 \mathrm{~mW} \mathrm{~cm}^{-2}$ for a walking subject at room temperature if a 
Table 5: Comparison of ideal $\eta_{\text {ideal }}$, total $\eta_{\text {total }}$ and measured total $\eta_{\text {meas }}$ efficiency for the energy conversion of human body heat to an electric storage element at $3.7 \mathrm{~V}$ in an indoor scenario.

\begin{tabular}{l|rrrrr} 
Approach & $\eta_{\text {ideal }}$ & $\eta_{\text {total }}$ & $\eta_{\text {meas }}$ & $\frac{\eta_{\text {meas }}}{\eta_{\text {ideal }}}$ & $\frac{\eta_{\text {meas }}}{\eta_{\text {total }}}$ \\
\hline pTEG & $1.96 \%$ & $0.057 \%$ & $0.014 \%$ & $0.71 \%$ & $24 \%$ \\
mTEG & $1.96 \%$ & $0.088 \%$ & $0.014 \%$ & $0.71 \%$ & $16 \%$ \\
\hline
\end{tabular}

heat sink with $K_{\operatorname{sink}}=29 \mathrm{~K} \mathrm{~W}^{-1}$ is applied. We can now compare the measured efficiency $\eta_{\text {meas }}$ in the real-world experiment (11) with the ideal efficiency $\eta_{\text {ideal }}$ (4) for $Z T \rightarrow \infty$ and with the calculated total efficiency $\eta_{\text {total }}$ (6) including perfect thermal and electric matching and DC-DC conversion, as shown in Table 5 . For the calculations, a constant core temperature of the lower arm of $35^{\circ} \mathrm{C}$ and a worst-case scenario $\left(\Delta T_{T E G}<1.75^{\circ} \mathrm{C}\right)$ is assumed: the subject sitting indoors without movement. Both harvesters achieve $0.71 \%$ of the ideal efficiency. According to (5) even small improvements of $Z T$ could raise this number significantly. The calculated total efficiency $\eta_{\text {total }}$ is $55 \%$ higher for the mTEG as a result of the high module figure of merit $Z T_{m}$ which is partly compensated for by the low electric efficiency $\eta_{e l}$. In the measurement, the $\mu$ TEG reaches $24 \%$ and the mTEG $16 \%$ of the total efficiency $\left(\eta_{\text {meas }} / \eta_{\text {total }}\right)$ resulting in equal output power. This is partly due to incomplete thermal matching of the used harvesters which is a consequence of high wearability (no stacking). Additionally, the LTC3108 DC-DC converter is not able to adapt to the load resistance of the mTEG, causing additional electric losses. A combination of increased $Z T$ values and electric conversion efficiency at low input voltages will enable higher efficiency in future devices. Although the total efficiency from body heat to an electric storage at $3.7 \mathrm{~V}$ is low, we will demonstrate that the acquired energy can be effectively applied to supply a wearable hardware platform.

\subsection{Application Case-Study}

After evaluating the achievable harvesting performance, we show in a case study that this harvesting power is sufficient to supply real applications. The example application considered here is a self-sustainable multi-sensor bracelet [49] that features various sensors that can be turned on and off independently for aggressive power management. A buck converter is used to supply the device efficiently for the large voltage difference of up to $2.2 \mathrm{~V}$ between battery and supply voltage. In combination with the buck converter's adjustable supply voltage, this allows minimizing the voltage and power consumption of the circuit. The power consumption numbers for the different operation modes of the bracelet and an optional Bluetooth Low Energy (BLE) transceiver are given in Table 6. For this case study, two harvesting scenarios are considered: an indoor office scenario without physical activity of the wearer, and an outdoor high activity scenario where the subject is moving. The real-world experiment showed that the harvesting power can be as low as $40 \mu \mathrm{W}$ in the indoor, while more than $800 \mu \mathrm{W}$ can be harvested in the outdoor scenario. 
Table 6: Power and energy requirements for the individual system components of the multisensor wearable application [49]. Bluetooth Low Energy (BLE) power for the transmission of one status update message with the CC2650 SoC [50].

\begin{tabular}{l|rrr} 
Subsystem & Power $[\mathrm{mW}]$ & Time $[\mathrm{ms}]$ & Energy [mJ] \\
\hline Accelerometer & 2.67 & 60000 & 160 \\
Microphone & 3.632 & 5000 & 18.16 \\
Camera & 3.77 & 47 & 0.177 \\
Display & 12.83 & 1192 & 15.298 \\
Status Transmission (BLE) & 11.31 & 3.12 & 0.35 \\
Deep Sleep & 0.0037 & - & - \\
\hline
\end{tabular}

The very low harvesting power of the indoor scenario, only very simplistic tasks like activity logging can be performed. The power budget of $40 \mu \mathrm{W}$ allows: sampling one second of audio data every two minutes, acquisition of one image every 40 seconds, and sending three status bytes via BLE every 40 seconds. These are only very few sensor data samples per hour, but for slowly changing low-activity scenarios the larger delay between sensor samples can be tolerated. In the outdoor scenario, the 20 times higher power budget allows more sophisticated applications, like the following example: sampling 5 seconds accelerometer data every 30 seconds, periodic image acquisition with a 5 second interval, recording 6 seconds of audio every two minutes, giving feedback to the user by updating the an e-ink display every two minutes, and sending a status update via BLE as often as every 5 seconds. The increased power budget results in a much higher functionality of the wearable and shows the potential of using body heat for these types of application.

There is a large range of power levels in between the two extreme cases considered in this case study and so does the service or functionality of the wearable. Because the device can turn on or off sensors on demand, there is a good potential for energy aware applications. A higher service is provided to the user when the power increases during active periods, while the system increases the sleep times between sensor acquisition when the user's activity is minimal. This correlates nicely with the use cases of activity tracking and context recognition and shows the potential of supplying smart wearables from body heat. Furthermore, a harvesting circuit could also be augmented with another energy source to support multi-source harvesting [51, 13]. In the best case, an alternative source like solar harvesting can complement the low input power for low temperature difference scenarios.

\section{Conclusions}

Power optimization for thermoelectric energy harvesters applied on the human body is highly application specific and requires consideration of the complete system from human body heat to usable electric energy. This work focused on the critical and often neglected interaction between TEG and electric DC-DC conversion circuit. Two harvesting approaches using different TEG and DC-DC 
architectures were compared and we found that a mTEG with low output voltage but high module figure of merit can perform equal or superior to a high voltage $\mu$ TEG in terms of total conversion efficiency and output power.

The mTEG approach delivered up to $65 \%$ higher output power per area in a laboratory setup and up to $15 \%$ in a real-world experiment with different usage scenarios and ambient temperatures. The mTEG achieves $16 \%$ percent of its individual maximum total efficiency compared to $24 \%$ percent for the $\mu$ TEG due to lacking load matching in the LTC3108. The optimized thermal harvesting systems based on off-the-shelf components provided sufficient power (on average $260 \mu \mathrm{W}$ ( $\mu$ TEG) to $280 \mu \mathrm{W}$ (mTEG)) to operate a state-of-the-art multi-sensor wearable. The unobtrusive harvesters with an area of $20 \mathrm{~cm}^{2}$ and a weight of $39 \mathrm{~g}$ ( $\mu$ TEG) to $41 \mathrm{~g}$ (mTEG) were comfortable to wear in environmental temperatures from $13^{\circ} \mathrm{C}$ to $37^{\circ} \mathrm{C}$.

The system design and partitioning of non-intrusive wearable devices will benefit from two developments: the progress in ultra-low power devices and the progress in battery development and/or efficient low-temperature-difference, thermally matched energy harvesters. Thermal energy harvesters can significantly extend the time of operation of (chargeable) batteries or even replace them. The application and use case determines whether exchange of batteries (e.g. once a month using a standard CR2032 coin cell with $220 \mathrm{mAh}$ in this studys scenario) is convenient and safe enough, or if thermal harvesting systems can enable the power supply of systems during a life-time of several years. The business case will decide, if energy harvesters, additional circuits and customized thermal matching will be economical and allow widespread application.

\section{Acknowledgments}

This work is evaluated by the Swiss National Science Foundation, and funded by the Nano-Tera.ch BodyPoweredSense project and by grant 157048: Transient Computing Systems, with Swiss Confederation financing.

\section{References}

[1] M. Billinghurst, D. Busse, Rapid Prototyping for Wearables: Concept Design and Development for Head- and Wrist-mounted Wearables (Smart Watches and Google Glass), in: Proceedings of the Ninth International Conference on Tangible, Embedded, and Embodied Interaction, TEI '15, ACM, 2015, pp. 505-508. doi:10.1145/2677199.2683592

[2] S. Li, L. D. Xu, S. Zhao, The internet of things: a survey, Information Systems Frontiers 17 (2) (2015) 243-259. doi:10.1007/s10796-014-9492-7

[3] M. Gorlatova, J. Sarik, G. Grebla, M. Cong, I. Kymissis, G. Zussman, Movers and Shakers: Kinetic Energy Harvesting for the Internet of Things, in: 2014 ACM International Conference on Measurement and Modeling of Computer Systems, SIGMETRICS '14, ACM Press, 2014, pp. 407-419. doi:10.1145/2591971.2591986. 
[4] J. Gubbi, R. Buyya, S. Marusic, M. Palaniswami, Internet of Things (IoT): A vision, architectural elements, and future directions, Future Generation Computer Systems 29 (7) (2013) 1645-1660. doi:10.1016/j.future. 2013.01.010.

[5] N. A. Bhatti, M. H. Alizai, A. A. Syed, L. Mottola, Energy Harvesting and Wireless Transfer in Sensor Network Applications, ACM Transactions on Sensor Networks 12 (3) (2016) 1-40. doi:10.1145/2915918.

[6] J. A. Paradiso, T. Starner, Energy scavenging for mobile and wireless electronics, IEEE Pervasive Computing 4 (1) (2005) 18-27. doi:10.1109/ MPRV.2005.9.

[7] P. Pillatsch, E. M. Yeatman, A. S. Holmes, A piezoelectric frequency upconverting energy harvester with rotating proof mass for human body applications, Sensors and Actuators A: Physical 206 (2014) 178-185. doi: 10.1016/j.sna.2013.10.003.

[8] T. Soyata, L. Copeland, W. Heinzelman, RF Energy Harvesting for Embedded Systems: A Survey of Tradeoffs and Methodology, IEEE Circuits and Systems Magazine 16 (1) (2016) 22-57. doi:10.1109/MCAS.2015.2510198.

[9] W. Glatz, E. Schwyter, L. Durrer, C. Hierold, Bi2Te3-Based Flexible Micro Thermoelectric Generator With Optimized Design, Journal of Microelectromechanical Systems 18 (3) (2009) 763-772. doi:10.1109/JMEMS.2009. 2021104 .

[10] Y. Shi, Y. Wang, Y. Deng, H. Gao, Z. Lin, W. Zhu, H. Ye, A novel selfpowered wireless temperature sensor based on thermoelectric generators, Energy Conversion and Management 80 (2014) 110-116. doi:10.1016/j. enconman.2014.01.010.

[11] V. Leonov, P. Fiorini, T. Torfs, R. J. M. Vullers, C. Van Hoof, Thermal matching of a thermoelectric energy harvester with the environment and its application in wearable self-powered wireless medical sensors, in: Thermal Investigations of ICs and Systems, IEEE, 2009, pp. 95-100.

[12] M. Lossec, B. Multon, H. Ben Ahmed, C. Goupil, Thermoelectric generator placed on the human body: system modeling and energy conversion improvements, European Physical Journal Applied Physics 52 (1) (2010) 10. doi:10.1051/epjap/2010121.

[13] A. S. Weddell, M. Magno, G. V. Merrett, D. Brunelli, B. M. Al-Hashimi, L. Benini, A Survey of Multi-Source Energy Harvesting Systems, in: Proceedings of the Conference on Design, Automation and Test in Europe, 2013, pp. 905-908. doi:10.7873/DATE.2013.190.

[14] P. Spies, M. Pollak, L. Mateu, Handbook of Energy Harvesting Power Supplies and Applications, Pan Stanford, 2015. doi:10.1201/b18523. 
[15] V. Leonov, Thermoelectric Energy Harvesting of Human Body Heat for Wearable Sensors, IEEE Sensors Journal 13 (6) (2013) 2284-2291. doi: 10.1109/JSEN. 2013.2252526.

[16] M. Magno, D. Porcarelli, D. Brunelli, L. Benini, InfiniTime: A Multisensor Energy Neutral Wearable Bracelet, in: 2014 International Green Computing Conference, 2014, pp. 1-8. doi:10.1109/IGCC.2014.7039180.

[17] B. Yang, C. Lee, W. Xiang, J. Xie, J. H. He, R. K. Kotlanka, S. P. Low, H. Feng, Electromagnetic energy harvesting from vibrations of multiple frequencies, Journal of Micromechanics and Microengineering 19 (3) (2009) 035001. URL http://stacks . iop.org/0960-1317/19/i=3/a=035001

[18] X. Cheng, B. Meng, X. Zhang, M. Han, Z. Su, H. Zhang, Wearable electrode-free triboelectric generator for harvesting biomechanical energy, Nano Energy 12 (2015) 19-25. doi:10.1016/j.nanoen.2014.12.009.

[19] N. Zhang, J. Chen, Y. Huang, W. Guo, J. Yang, J. Du, X. Fan, C. Tao, A Wearable All-Solid Photovoltaic Textile, Advanced Materials 28 (2) (2016) 263-269. doi:10.1002/adma.201504137.

[20] Y. Li, L. Meng, Y. M. Yang, G. Xu, Z. Hong, Q. Chen, J. You, G. Li, Y. Yang, Y. Li, High-efficiency robust perovskite solar cells on ultrathin

n flexible substrates, Nature Communications 7 (2016) 10214. doi:10.1038/ ncomms 10214,

[21] A. K. Ermeey, A. P. Hu, M. Biglari-Abhari, K. C. Aw, Indoor 2.45 GHz WiFi Energy Harvester With Bridgeless Converter, IEEE Journal on Selected

घ. Areas in Communications 34 (5) (2016) 1536-1549. doi:10.1109/JSAC. 2016.2551578

[22] T. Starner, Human-powered wearable computing, IBM Systems Journal 35 (3.4) (1996) 618-629. doi:10.1147/sj.353.0618.

[23] M. Lossec, B. Multon, H. Ben Ahmed, Sizing optimization of a thermoelectric generator set with heatsink for harvesting human body heat, Energy Conversion and Management 68 (2013) 260-265. doi:10.1016/j. enconman.2013.01.021.

[24] D. C. Hoang, Y. K. Tan, H. B. Chng, S. K. Panda, Thermal energy harvesting from human warmth for wireless body area network in medical healthcare system, in: 2009 International Conference on Power Electronics and Drive Systems (PEDS), IEEE, 2009, pp. 1277-1282. doi: 10.1109/PEDS.2009.5385814.

[25] M. Van Bavel, V. Leonov, R. F. Yazicioglu, T. Torfs, C. Van Hoof, N. Posthuma, R. Vullers, Wearable battery-free wireless 2-channel EEG systems powerd by energy scavengers, Sensors \& Transducers Journal 94 (7) (2008) 103-115. 
[26] M. Wahbah, M. Alhawari, B. Mohammad, H. Saleh, M. Ismail, Characterization of Human Body-Based Thermal and Vibration Energy Harvesting for Wearable Devices, IEEE Journal on Emerging and Selected Topics in Circuits and Systems 4 (3) (2014) 354-363. doi:10.1109/JETCAS.2014. 2337195 .

[27] M.-K. Kim, M.-S. Kim, S. Lee, C. Kim, Y.-J. Kim, Wearable thermoelectric generator for harvesting human body heat energy, Smart Materials and Structures 23 (10) (2014) 105002. doi:10.1088/0964-1726/23/10/ 105002

[28] Z. Lu, M. Layani, X. Zhao, L. P. Tan, T. Sun, S. Fan, Q. Yan, S. Magdassi, H. H. Hng, Fabrication of Flexible Thermoelectric Thin Film Devices by Inkjet Printing, Small 10 (17) (2014) 3551-3554. doi:10.1002/smll. 201303126.

[29] S. J. Kim, J. H. We, B. J. Cho, A wearable thermoelectric generator fabricated on a glass fabric, Energy and Environmental Science 7 (6) (2014) 1959-1965. doi:10.1039/C4EE00242C

[30] J. Chen, L. Zuo, Y. Wu, J. Klein, Modeling, experiments and optimization of an on-pipe thermoelectric generator, Energy Conversion and Management 122 (2016) 298-309. doi:10.1016/j.enconman.2016.05.087.

[31] J.-H. Meng, X.-D. Wang, W.-H. Chen, Performance investigation and design optimization of a thermoelectric generator applied in automobile exhaust waste heat recovery, Energy Conversion and Management 120 (2016) 71-80. doi:10.1016/j.enconman.2016.04.080.

[32] E. J. Carlson, K. Strunz, B. P. Otis, A $20 \mathrm{mV}$ input boost converter with efficient digital control for thermoelectric energy harvesting, IEEE Journal of Solid-State Circuits 45 (4) (2010) 741-750. doi:10.1109/JSSC. 2010. 2042251.

[33] S. Bandyopadhyay, A. P. Chandrakasan, Platform architecture for solar, thermal, and vibration energy combining with MPPT and single inductor,

n IEEE Journal of Solid-State Circuits 47 (9) (2012) 2199-2215. doi:10. 1109/JSSC. 2012.2197239.

[34] Y. K. Ramadass, A. P. Chandrakasan, A batteryless thermoelectric energyharvesting interface circuit with $35 \mathrm{mV}$ startup voltage, in: 2010 IEEE International Solid-State Circuits Conference (ISSCC), 2010, pp. 486-487. doi:10.1109/ISSCC.2010.5433835.

[35] M. P. Andersen, G. Fierro, D. E. Culler, System Design for a Synergistic, Low Power Mote/BLE Embedded Platform, in: 2016 15th ACM/IEEE International Conference on Information Processing in Sensor Networks, 2016, pp. 1-12. doi:10.1109/IPSN.2016.7460722. 
[36] C. Zhou, C. Tu, J. Tian, J. Feng, Y. Gao, X. Ye, A low power miniaturized monitoring system of six human physiological parameters based on wearable body sensor network, Sensor Review 35 (2) (2015) 210-218. doi:10.1108/sr-08-2014-687.

[37] C. Wang, W. Lu, M. R. Narayanan, S. J. Redmond, N. H. Lovell, Lowpower technologies for wearable telecare and telehealth systems: A review, Biomedical Engineering Letters 5 (1) (2015) 1-9. doi:10.1007/ s13534-015-0174-2.

[38] M. Strasser, R. Aigner, M. Franosch, G. Wachutka, Miniaturized thermoelectric generators based on poly-Si and poly-SiGe surface micromachining, Sensors and Actuators A: Physical 97 (2002) 535-542. doi: 10.1007/978-3-642-59497-7_5.

[39] H. J. Goldsmid, Conversion efficiency and figure-of-merit, CRC Handbook of Thermoelectrics (1995) 19-25.

[40] F. L. Curzon, B. Ahlborn, Efficiency of a Carnot engine at maximum power output, American Journal of Physics 43 (1) (1975) 22-24. doi:10.1119/ 1.10023 .

[41] L. E. Bell, Alternate thermoelectric thermodynamic cycles with improved power generation efficiencies, in: Thermoelectrics, 2003 Twenty-Second International Conference on - ICT, IEEE, 2003, pp. 558-562. doi:10.1109/ ict.2003.1287574.

[42] O. Brand, G. K. Fedder, C. Hierold, J. G. Korvink, O. Tabata, D. Briand, E. Yeatman, S. Roundy, Micro Thermoelectric Generators, in: Micro Energy Harvesting, John Wiley \& Sons, 2015, Ch. 12, pp. 245-269. doi: $10.1002 / 9783527672943$.

[43] J. W. Stevens, Optimal design of small $\Delta \mathrm{T}$ thermoelectric generation systems, Energy Conversion and Management 42 (6) (2001) 709-720. doi:10.1016/S0196-8904(00)00099-6.

[44] M. Freunek, Untersuchung der Thermoelektrik zur Energieversorgung autarker Systeme, in: Untersuchung der Thermoelektrik zur Energieversorgung autarker Systeme, Der Andere Verlag, 2010, Ch. 4, pp. 67-130.

[45] Texas Insturments, bq25504 Ultra Low-Power Boost Converter With Battery Management for Energy Harvester Applications (2015). URL http://www.ti.com/lit/ds/symlink/bq25504.pdf

[46] Linear Technology, LTC3108 Ultralow Voltage Step-Up Converter and Power Manager (2013).

URL http://cds.linear.com/docs/en/datasheet/3108fc.pdf 
[47] A. Gomez, L. Sigrist, M. Magno, L. Benini, L. Thiele, Dynamic Energy Burst Scaling for Transiently Powered Systems, in: Proceedings of the Conference on Design, Automation and Test in Europe, 2016, pp. 349-354. doi:10.3850/9783981537079_0403

[48] M. Thielen, P. Streit, M. Muoth, W. Glatz, C. Hierold, Thermoelectric Energy Harvesting for autonomous Body Sensor Networks, Nano-Tera Annual Plenary Meeting (2014).

URL www .nano-tera.ch/pdf/posters2014/BodyPoweredSenSE239.pdf

[49] M. Magno, D. Brunelli, L. Sigrist, R. Andri, L. Cavigelli, A. Gomez, L. Benini, InfiniTime: Multi-Sensor Wearable Bracelet with Human Body Harvesting, Sustainable Computing: Informatics and Systems 11 (2016) 38-49. doi:10.1016/j.suscom.2016.05.003

[50] Texas Insturments, Application Report: Measuring Bluetooth Smart Power Consumption (2016).

URL http://www.ti.com/lit/an/swra478a/swra478a.pdf

[51] D. Carli, D. Brunelli, L. Benini, M. Ruggeri, An effective multi-source energy harvester for low power applications, in: Proceedings of the Conference on Design, Automation and Test in Europe, 2011, pp. 1-6. doi: 10.1109/DATE.2011.5763142. 